\title{
Genetic Variation for Nine Autosomal Short Tandem Repeat (STRs) in
} Egyptian Population

\section{Tarek Tahaa ${ }^{1}$, Sagy Elzalabanyb ${ }^{2}$, Olfat Shaker ${ }^{3^{*}}$ and Sahar Fawz}

${ }^{1}$ Department of Immunology, Egyptian Armed Forces Central Labs, Egypt

${ }^{2}$ Main Biomedical Engineering Workshop, Egypt

${ }^{3}$ Department of Medical Biochemistry and Molecular Biology, Egypt

${ }^{4}$ Department of Systems and Biomedical Engineering, Egypt

*Corresponding author: Olfat Shaker, Department of Medical Biochemistry and Molecular Biology, Faculty of Medicine, Cairo University, Egypt, Tel: +20 2 35676105; Email: olfatshaker@yahoo.com

Received date: April 9, 2018; Accepted date: April 16, 2018; Published date: April 23, 2018

Copyright: (c) 2018 Tahaa T, et al. This is an open-access article distributed under the terms of the Creative Commons Attribution License, which permits unrestricted use, distribution, and reproduction in any medium, provided the original author and source are credited.

\begin{abstract}
Allele frequencies for 9 autosomal short tandem repeat loci (STRs) D3S1358, VWA, FGA, THO1, TPOX, CSF1PO, D5S818, D13S317, D7S820 and a locus allowing for sex discrimination were determined in 1000 unrelated Egyptians. Blood samples were collected after obtaining an informed consent. The DNA was extracted by using the standard phenol-chloroform extraction method and then amplified using commercial available kit (AmpF $\mathrm{L}$ STR profiler kit, Applied Bio systems) by the polymerase chain reaction, and subsequently typed using capillary electrophoresis (3130X1 Genetic Analyser, Applied Bio systems). All loci met Hardy-Weinberg expectations and no mutations were found among subjects.
\end{abstract}

Keywords: DNA; STRs; Allele frequency; Egyptian

\section{Introduction}

Deoxyribonucleic acid (DNA) typing is widely used for personal identification and knowing the source of biological samples found in the crime scene [1]. In view of their high level of variability, autosomal short tandem repeats (STRs) are very useful as markers of forensic and Population genetics studies [2].

STRs loci have repeat units of 3-5 bp and allele sizes of 100-300 bp. Some loci have alleles differing by a number of complete repeat units, whereas others display complex polymorphism with some alleles differing in size by only 1 bp [3].

Federal Bureau of Investigation has recommended using 13 STR loci, known as CODIS 13, as the loci of choice for forensic use. This recommendation has been accepted by forensic laboratories all over the world, thus the DNA typing can be compared to each other [4].

\section{Population}

Blood samples were taken from 1000 unrelated samples form Egyptian Populations.

\section{Methodology}

\section{DNA extraction}

DNA was isolated from whole EDTA blood using (QiA amp DNA blood by Qiagen-Gmph). Genomic DNA was extracted through phenol-chloroform extraction procedure and amplified using Amp FISTR identifiler ${ }^{\text {mo }}$ and Amp FISTR Yfiler ${ }^{\text {mo }}$ kits. Genotyping was performed on ABI 3130 Genetic Analyzer and the data was analysed using Gene Mapper ID v4 software. Statistical evaluation was done.
The peak height threshold was 50 RFU for heterozygous and $200 \mathrm{RFU}$ for homozygous alleles. All steps were followed using kit instructions.

\section{PCR amplification}

The samples were amplified in single reactions for all 9 loci using fluorescently labelled primers.

\section{Typing}

Separation made by capillary electrophoresis in an ABI 3130 Genetic Analyser using the separation medium Performance Optimized Polymer (POP) 7 and $36 \mathrm{~cm}$ capillaries array (Applied Bio systems). The LIZ labelled ladder (Gene Scan 500 LIZ) was used for sizing the determinations of the amplified fragments in combination with Gene Mapper ID v4 (Applied Bio systems). Allelic calls and genotyping carried out by comparison to the reference allelic ladder included in the kit, using Gene Mapper ID v4 (Applied Bio systems).

\section{Results}

The results for the 9 STRs are presented in Table 1.

\section{Discussion}

The frequency of each allele for each locus and the observed heterozygosity (Ho) was calculated from the number of observed genotypes in the sample (direct gene counting). Expected heterozygosity $(\mathrm{He})$ was estimated as $1 \_\mathrm{Pi}$ p2i where pi is the frequency of the ith allele in the locus. Microsoft Excel-Power status program (Promega cooperation) [5] http://www.promega.com/ geneticid tools/powerstats to calculate: [Match probability (MP), Typical paternity index (TPI), Polymorphism information content 
Citation: Tahaa T, Elzalabanyb S, Shaker O, Fawz S (2018) Genetic Variation for Nine Autosomal Short Tandem Repeat (STRs) in Egyptian Population. J Forensic Med 3: 123. doi:10.4172/2472-1026.1000123

Page 2 of 4

(PIC), Power of exclus and Power of discrimination (PD)] was used for calculation of the results.

SPSS test (Pearson's chi-square test) was used to calculate HardyWeinberg equilibrium followed by correction using Bonferroni Correction [6]. In addition, two sided tests for LD were used to measures P-value to calculate the linkage equilibrium (result were considered significant when $\mathrm{P}<0.05$, highly significant $\mathrm{P}<0.01$ and externally significant when $\mathrm{P}<0.001)$. Finally, FST genetic distance was used to calculate locus by locus using Arlequin program to measure $\mathrm{F}$ statistics (for inbreeding coefficient) [7].

\begin{tabular}{|c|c|c|c|c|c|c|c|c|c|}
\hline Allele & D3S1358 & vWA & FGA & TH01 & TPOX & CSF1PO & D5S818 & D13S317 & D7S820 \\
\hline 5 & - & - & - & 0.002 & - & - & - & - & - \\
\hline 5.3 & - & - & - & 0.001 & - & - & - & - & - \\
\hline 6 & - & - & - & 0.222 & 0.012 & - & - & - & 0.001 \\
\hline 6.3 & - & - & - & 0.001 & - & - & - & - & - \\
\hline 7 & - & - & - & 0.2 & 0.004 & 0.005 & - & - & 0.017 \\
\hline 8 & - & - & - & 0.118 & 0.472 & 0.015 & 0.03 & 0.133 & 0.162 \\
\hline 8.3 & - & - & - & 0.005 & - & - & - & - & - \\
\hline 9 & - & - & - & 0.332 & 0.175 & 0.022 & 0.078 & 0.066 & 0.094 \\
\hline 9.2 & - & - & - & - & - & - & - & - & 0.001 \\
\hline 9.3 & - & - & - & 0.085 & 0.001 & - & - & - & - \\
\hline 10 & - & - & - & 0.037 & 0.087 & 0.284 & 0.115 & 0.047 & 0.316 \\
\hline 11 & - & 0.001 & - & - & 0.231 & 0.301 & 0.246 & 0.273 & 0.231 \\
\hline 12 & 0.002 & $22-$ & - & - & 0.02 & 0.307 & 0.332 & 0.349 & 0.148 \\
\hline 13 & 0.006 & 0.004 & - & - & 0.001 & 0.06 & 0.184 & 0.109 & 0.031 \\
\hline 14 & 0.059 & 0.075 & - & - & - & 0.008 & 0.014 & 22 & 0.002 \\
\hline 15 & 0.264 & 0.137 & - & - & - & 0.001 & 0.002 & 0.003 & - \\
\hline 16 & 0.273 & 0.246 & - & - & - & - & 0.001 & - & - \\
\hline 17 & 0.251 & 0.271 & - & - & - & - & - & - & - \\
\hline 18 & 0.137 & 0.183 & 0.009 & - & - & - & - & - & - \\
\hline 18.2 & - & - & 0.001 & - & - & - & - & - & - \\
\hline 19 & 0.01 & 0.073 & 0.062 & - & - & - & - & - & - \\
\hline 20 & - & 0.01 & 0.09 & - & - & - & - & - & - \\
\hline 20.2 & - & - & 0.001 & - & - & - & - & - & - \\
\hline 21 & - & 0.003 & 0.134 & - & - & - & - & - & - \\
\hline 21.2 & - & - & 0.001 & - & - & - & - & - & - \\
\hline 22 & - & - & 0.163 & - & - & - & - & - & - \\
\hline 22.2 & - & - & 0.003 & - & - & - & - & - & - \\
\hline 23 & - & - & 0.177 & - & - & - & - & - & - \\
\hline 23.2 & - & - & 0.002 & - & - & - & - & - & - \\
\hline 24 & - & - & 0.169 & - & - & - & - & - & - \\
\hline 24.2 & - & - & 0.001 & - & - & - & - & - & - \\
\hline 25 & - & - & 0.107 & - & - & - & - & - & - \\
\hline
\end{tabular}




\begin{tabular}{|l|l|l|l|l|l|l|l|l|l|}
\hline 26 & - & - & 0.043 & - & - & - & - & - \\
\hline 27 & - & - & 0.021 & - & - & - & - \\
\hline 28 & - & - & 0.014 & - & - & - & - & - \\
\hline 29 & - & - & 0.005 & - & - & - & - & - & - \\
\hline 30 & - & - & 0.002 & - & - & - & - & - & - \\
\hline H1 & 0.775 & 0.806 & 0.834 & 0.672 & 0.663 & 0.727 & 0.753 & 0.758 & 0.7 \\
\hline H2 & 0.225 & 0.195 & 0.1665 & 0.328 & 0.337 & 0.273 & 0.247 & 0.242 & 0.233 \\
\hline PD & 0.902 & 0.923 & 0.965 & 0.89 & 0.853 & 0.872 & 0.907 & 0.91 & 0.97 \\
\hline PE & 0.522 & 0.602 & 0.773 & 0.447 & 0.39 & 0.463 & 0.562 & 0.592 \\
\hline P & 0.391 & 0.12 & 0.601 & 0.86 & 0 & 0.401 & 0.841 & 0.98 & 0.581 \\
\hline MP & 0.11 & 0.073 & 0.031 & 0.092 & 0.14 & 0.13 & 0.091 & 0.071 \\
\hline PI & 0.74 & 0.756 & 0.863 & 0.713 & 0.621 & 0.672 & 0.741 & 0.92 \\
\hline Typical PI & 2.2 & 1.8 & 2.17 & 2.57 & 1.43 & 1.97 & 2.19 & 1.55 \\
\hline
\end{tabular}

Table 1: Description of 9 STRs. H1: Observed Heterozygosity; H2: Observed Homozygosity; PD: Power of Discrimination; PE: Power of Exclusion P: Probability Values of Exact for Hardy-Weinberg Equilibrium; MP: Matching Probability; PI: Polymorphism Information Content; Typical PI: Typical Paternity Index.

The results that have been drawn from the present study include that the number of different alleles observed across the Egyptian Population was found to be 92 alleles with a predicated number of $3.7332 \times 10^{\wedge} 15$ possible genotypes. In addition, the highest allele frequencies occurred in the allele 8 for TPOX locus (0.472) and allele 12 for D13S317 (0.349). The most common alleles at the other seven loci were allele 16 for D3S1358 (0.273), allele 9 for TH01 (0.332), allele 12 for D5S818 (0.332), allele 10 for D7S820 (0.316), allele 12 for CSF1PO (0.307), allele 17 for VWA (0.271) and allele 23 for FGA (0.177). While the FGA locus showed the largest number of different alleles (19 alleles) and D3S1358 and D13S317 locus represented the smallest number of different alleles (8 alleles). Results also revealed that the heterozygosity of the nine STR loci ranged from $62 \%$ to $86 \%$ (mean value $74 \%$ ), the locus with the highest heterozygosity was FGA (83.4\%), while locus TPOX has the lowest heterozygosity (66.3\%).

The power of discrimination values for all tested loci was above $84 \%$ for the CSF1PO and TPOX loci, from $90 \%$ to $96 \%$ for TH01, D13S317, D3S1358, D7S820, VWA, FGA and D5S818. Tthe locus with the highest discrimination power was FGA (0.965), while locus TPOX has the lowest discrimination power (0.853). The locus with the highest Power of exclusion was FGA (0.773), while locus TPOX has the lowest Power of exclusion (0.390). All loci are highly polymorphic (PIC over $0.5)$ [8]. Locus with the highest polymorphism information content was FGA (0.863), while locus TPOX has the lowest polymorphism information content (0.621). In addition, the locus with the highest Matching probability was FGA (0.31), while locus TPOX has the lowest Matching probability (0.14).

None of the tested loci showed significant departure for HWE except TPOX, so, they are statistically independent. For locus TPOX, an excess of homozygotes substantially more often observed than expected. Other reasons for the deviation observed at the TPOX locus include inbreeding, Population substructure, selection, or "silent" alleles [9].
The combined probability of exclusion, power of discrimination, probability of matching value for all the 9 STR loci were 0.999875 , $1.436 \times 10^{\wedge}-11$ and $1.7 \times 10^{\wedge}-10$, respectively. Also, frequencies from the two most common alleles at each of the 9 loci were used to estimate a theoretical most common STR profile, which occurs with a combined frequency of approximately one person in $3.36 \times 10^{\wedge} 7$.

There was no evidence of departure from independence of alleles between all tested loci (linkage equilibrium). The D5S818 and CSF1PO, was in linkage equilibrium ( $\mathrm{P}$-value $=0.46596$ ). The FIS value for Egyptian Population has been calculated as $(-0.036)$ with nonsignificant P-value of (0.705). The low FIS value indicates low level of inbreeding within the Population and that the Population is in random mating with a high level of heterozygosity. The arrangement of the polymorphic markers in a decreasing order was FGA, VWA, D7S820, THO1, D5S818, CSF1PO, D13S317 and D3S1358, TPOX.

The results indicate that the examined 9 STR loci are useful genetic markers for forensic personal identification and paternity testing in the Egyptian Population.

Previously published article was done on Egyptian Population [10-14]. Also, Coudray et al. [15], studied alleles' frequency of 15 STRs in three Egyptian Populations of different ethnic groups (Berbers, Muslims and Copts). They stated that six alleles (8.9.10.11,12 and 13) were detected at CSF1PO locus in Egyptian Berbers. They stated that the most frequent allele was allele $10(0.291)$ while the least frequent was allele 9 (0.005). In Egyptian Muslims from Adaima, 8 alleles (8, 9, $10,10.2,11,12,13$ and 14) were detected at CSF1PO locus. Allele 10 also was the most frequent allele $(0.31)$ while the least were alleles 8 and 10.2 (0.005 for each). In the same village, alleles' frequency of Egyptian Copts showed that 7 alleles (8, 9. 10, 11, 12, 13 and 15) were detected at CSF1PO locus. The most frequent allele was allele 12 $(0.385)$ then allele $10(0.365)$ while the least was allele $15(0.005)$. 
Citation: Tahaa T, Elzalabanyb S, Shaker O, Fawz S (2018) Genetic Variation for Nine Autosomal Short Tandem Repeat (STRs) in Egyptian Population. J Forensic Med 3: 123. doi:10.4172/2472-1026.1000123

Page 4 of 4

\section{Conclusion}

The results of this study will add to the data bank of various studies conducted on the Egyptian Population. With respect to the distribution of alleles at each STR locus, the loci were found to be substantially polymorphic in this Population indicating good credibility of 9 STRs markers.

\section{Acknowledgement}

This work was carried out in Egyptian army central labs.

\section{References}

1. Atmadja DS (1989) DNA fingerprint: A new method on disputed paternity. Indonesian J Pathol 3: 1-6.

2. El Ossmani H, Bouchrif B, Aboukhalid R, Bouabdillah M, Gazzaz B, et al. (2010) Assessment of phylogenetic structure of Berber-speaking Population of Azrou using 15 STRs of Identifiler kit. Leg Med 12: 52-56.

3. Delghandi M, Branting BJ, Jørgensen L (2001) Evaluation of a quadruplex short atandem repeat system (HUMVWA31/A, HUMD11S554, HUMAPOAI1 and HUMACTBP2 loci) for forensic identity testing, confident typing of complex alleles and Population databases. Croat Med J 42: 33-44.

4. Untoro E, Atmadja DS, Pu CE, Wu FC (2009) Allele frequency of CODIS 13 in Indonesian Population. Leg Med 11: S203-S205.

5. Tereba A (1999) Tools for analysis of Population statistics. Profiles DNA 2: $14-16$.

6. Weir BS (1990) Multiple tests. In: Genetic Data Analysis II. Sinauer Associates, USA.
7. Schneider S, Roessli D, Excoffier V. Arlequin D (2000) A software for Population genetics data analysis. Ver. 2.0, Genetics and Biometry Laboratory, Department of Anthropology and Ecology, University of Geneva, Geneva, Switzerland,

8. Elmors DA, Elbakry AA (2009) Alleles frequency distribution of 2 STR loci in Egyptian Population. Mansoura J Forensic Med Clin Toxicol 17.

9. (1996) National Research Council, the Evaluation of Forensic DNA Evidence, National Academy Press, Washington DC.

10. Tahir MA, Herrera RJ, el-Gohary M, Granoff M, Amjad M (2003) Allele frequency distribution of Power Plex 1.2 and Profiler Plus short tandem repeats (STR) loci in Egyptian Population. J Forensic Sci 48: 889-890.

11. Ahmed A, Linacre AM, Mohammed AA, Vanezis P, Goodwin W (2001) STR Population data for 10 STR loci including the Gene Print Power-Plex 1.2 kit from El-Minia (Central Egypt). Forensic Sci Int 117: 233-234.

12. Klintschar M, Al-Hammadi N, Reichenpfader B (1999) Population genetic studies on the tetrameric short tandem repeat loci D3S1358, VWA, FGA, D8S1179, D21S11, D18S51, D5S818, D13S317 and D7S820 in Egypt. Forensic Sci Int 104: 23-31.

13. Coudray C, Guikard E, El-Shennawiallele F (2007) Frequency of 15 short tandem repeat (STRs) in 3 Egyptian Population of different ethenic groups. Forensic Sci Int 169: 260-265.

14. Omran GA, Rutty GN, Jobling MA (2009) Genetic variation of 15 autosomal STR loci in upper (Southern) Egyptians. Forensic Sci Int Genet 3: 39-44.

15. Coudray C, Guitard E, el-Chennawi F, Larrouy G, Dugoujon JM (2007) Allele frequencies of 15 short tandem repeats (STRs) in three Egyptian Populations of different ethnic groups. Forensic Sci Int 4: 260-265. 